\title{
Space Charge Formation in LDPE/MgO Nano-composite Film under Ultra-high DC Electric Stress
}

\author{
Yuji Hayase Student Member (Musashi Institute of Technology) \\ Hiroyuki Aoyama Non-member (Musashi Institute of Technology) \\ Kohei Matsui Non-member (Musashi Institute of Technology) \\ Yasuhiro Tanaka Member (Musashi Institute of Technology) \\ Tatsuo Takada Member (Musashi Institute of Technology) \\ Yoshinao Murata Member (J-power Systems Corp.)
}

Keywords : nano-composite, low-density polyethylene (LDPE), magnesium oxide (MgO), space charge, pulsed electro-acoustic ( PEA) method

Space charge characteristics under a high DC electric field of more than $100 \mathrm{kV} / \mathrm{mm}$ in a nano-composite film of the low-density polyethylene (LDPE) mixed with a nano-size filler of $\mathrm{MgO}$ were studied using a pulsed electro-acoustic (PEA) method. To measure the space charge distribution using the PEA system under the high electric field in a thin film, a spatial resolution of the PEA system was improved. Using the improved system, the time dependence of the space charge formation in LDPE and LDPE/MgO nano-composite under the high electric field was observed. Figure 1 (a) shows a typical measurement result of a time dependent space charge distributions in LDPE under an average electric field of $250 \mathrm{kV} / \mathrm{mm}$ for 12 hours. It is clearly shown that a huge amount of a positive packet-like charge is injected from the anode into LDPE and it moves towards the cathode side with increasing its amount of charge. However, the movement gradually becomes slow down and finally it seems to be a stable state at 12 hours later from voltage on. Figure 1 (b) shows the electric field distributions which are obtained by calculating the integration of the space charge distributions shown in Fig. 1 (a). It is found that the electric field near the cathode is increasing with increase of the packet-like positive charge. Judging from Fig. 1 (b), it is clear that the maximum local electric field is gradually saturated to $470 \mathrm{kV} / \mathrm{mm}$. On the other hand, there is almost no such huge packet-like charge was observed in $\mathrm{LDPE} / \mathrm{MgO}$ with $\mathrm{MgO}$ content of more than $0.5 \mathrm{phr}$ while it was observed in that with $\mathrm{MgO}$ content of $0.2 \mathrm{phr}$. It is found that the generation of the packet-like charge under the high electric field depends on the content of $\mathrm{MgO}$ in LDPE/MgO nano-composite. Figure 2 shows a relationship between the initial applied and the local maximum electric fields in samples with various content of $\mathrm{MgO}$. In this figure, the broken line indicates the applied electric field. Therefore, the data over the line mean that the electric fields are enhanced by the space charge in the bulk. When the initial electric field of more than $100 \mathrm{kV} / \mathrm{mm}$ is applied to LDPE film, the local electric field is strongly enhanced. Especially in the case of more than $200 \mathrm{kV} / \mathrm{mm}$, the enhanced electric field reaches to about $500 \mathrm{kV} / \mathrm{mm}$. On the other hand, the enhancement of the local electric field in LDPE/MgO is very small compared with those in LDPE. Especially in the case of LDPE/MgO with $\mathrm{MgO}$ content of more than $0.5 \mathrm{phr}$, the enhancement is tiny even under $250 \mathrm{kV} / \mathrm{mm}$ of initial applied field. It is found that the generation of the packet-like charge under the high electric field strongly depends on the content of $\mathrm{MgO}$ in
LDPE/MgO nano-composite. In other words, it is found that even such a small amount of $\mathrm{MgO}$ content of $0.5 \mathrm{phr}$ in the nano-composite is effective to prevent the charge injection which is caused of the enhancement of the local electric field.

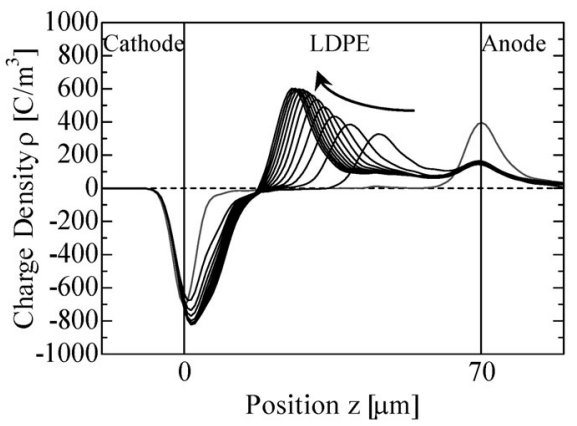

(a) Charge distribution

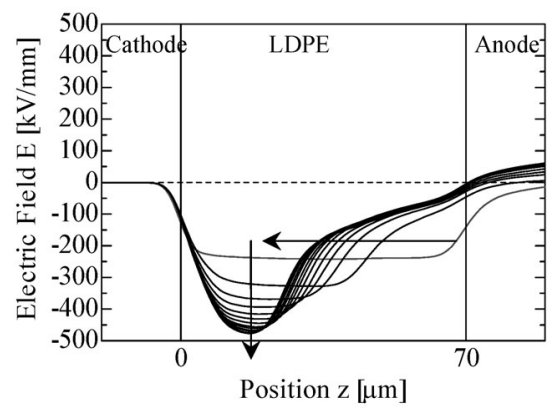

(b) Electric field distribution

Fig. 1. Time dependent (a) charge and (b) electric field distributions in LDPE under electric field of $250 \mathrm{kV} / \mathrm{mm}$ DC stress

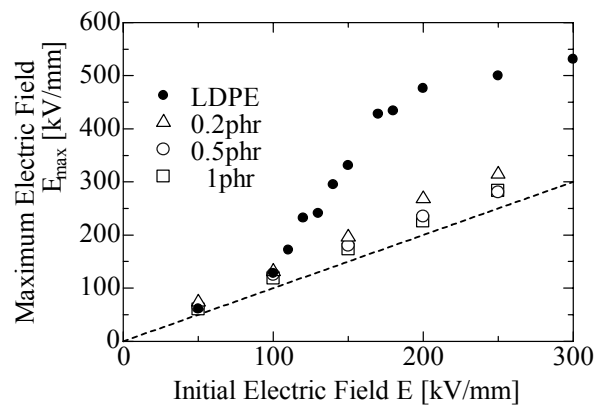

Fig. 2. Relationship between initial and local maximum electric fields 


\section{Space Charge Formation in LDPE/MgO Nano-composite Film under Ultra-high DC Electric Stress}

$\begin{array}{ll}\text { Yuji Hayase* } & \text { Student Member } \\ \text { Hiroyuki Aoyama* } & \text { Non-member } \\ \text { Kohei Matsui* }^{*} & \text { Non-member } \\ \text { Yasuhiro Tanaka* }^{*} & \text { Member } \\ \text { Tatsuo Takada* }^{*} & \text { Member } \\ \text { Yoshinao Murata }^{*} & \text { Member }\end{array}$

Space charge characteristics under high DC electric field of more than $100 \mathrm{kV} / \mathrm{mm}$ in a nano-composite film of the low density polyethylene (LDPE) mixed with a nano-size filler of $\mathrm{MgO}$ were studied using a pulsed electro-acoustic (PEA) method. To measure the space charge distribution using the PEA system under the high electric field in a thin film, a spatial resolution of the PEA system was improved. Using the improved system, the time dependence of space charge formation in LDPE and $\mathrm{LDPE} / \mathrm{MgO}$ nano-composite under the high electric field was observed. It is found that a huge amount of packet-like charge generation in LDPE makes a local electric field in the bulk higher. On the other hand, there is almost no such huge packet-like charge observed in LDPE/MgO with $\mathrm{MgO}$ content of more than $0.5 \mathrm{phr}$, while it was observed in that of $0.2 \mathrm{phr}$. It is found that the generation of the packet-like charge under the high electric field strongly depends on the content of $\mathrm{MgO}$ in the LDPE/MgO nano-composite.

Keywords : nano-composite, low-density polyethylene (LDPE), magnesium oxide (MgO), space charge, pulsed electro-acoustic (PEA) method

\section{Introduction}

Recently, the nano-composite insulating materials, which are made of base resin mixed with nano-size filler, are remarked as which show a good performance under high electric stress. The LDPE (low-density polyethylene) / MgO (magnesium oxide) nano-composite material, which is made of LDPE mixed with an $\mathrm{MgO}$ nano-filler has a higher resistivity under high DC stress than that in $\mathrm{LDPE}^{(1)}$. However, the mechanism of the improvement of the resistivity in LDPE/MgO has not been clear yet. It is thought that the resistivity against the DC stress is sometimes strongly affected by the space charge formation in the bulk of the sample. On the other hand, we have investigated a space charge formation in LDPE under ultra-high electric field of more than $100 \mathrm{kV} / \mathrm{mm}$ using the PEA (pulsed electro-acoustic) measurement system ${ }^{(2)}$. In the paper, we reported that a huge positive packet-like charge injected into LDPE increased the local electric field and it strongly affected to the breakdown occurred in LDPE under DC stress. In this paper, we tried to observe the space charge formation in LDPE/MgO nano-composite under such ultra-high DC electric field to investigate the relationship between the breakdown and space charge formation. To observe the space charge behavior in the sample under ultra-high electric field more than $100 \mathrm{kV} / \mathrm{mm}$, it was necessary to use a PEA system that was specially designed for the application of a high voltage such as more than $50 \mathrm{kV}$ to the sample. Since it is inconvenient to treat such a high voltage source in ordinary laboratory, we tried to develop a new system

\footnotetext{
* Musashi Institute of Technology

1-28-1, Tamazutsumi, Setagaya-ku, Tokyo 158-5587

** J-power Systems Corp.

5-1-1, Hitaka-cho, Hitachi 319-1414
}

with high spatial resolution to reduce the applying voltage for such an ultra-high electric field. At first, in this paper, we will introduce about the newly developed high-resolution system briefly. Then, we will show the measured space charge formation in LDPE and LDPE/MgO nano-composite under the ultra-high DC stress using the developed system.

\section{Development of PEA Measurement System with High Spatial Resolution}

In our previous report, we showed some measurement results of space charge behavior in LDPE under high electric field of more than $100 \mathrm{kV} / \mathrm{mm}^{(2)}$. However, the used system in the report was specially improved one for the high voltage application of maximum $60 \mathrm{kV}$. Since it is inconvenient to use such a high voltage power supply, we develop a new PEA apparatus with a high spatial resolution. If we can have a PEA measurement system with a good spatial resolution, we don't need to apply such high voltage to the sample any more. For example, when we apply the average electric field of $300 \mathrm{kV} / \mathrm{mm}$ to a sample of 50 $\mu \mathrm{m}$, it is necessary to apply only $15 \mathrm{kV}$ to the sample. Since an ordinary PEA apparatus has a spatial resolution of about $10 \mu \mathrm{m}$, however, the measurement result on such thin sample obtained using the ordinary apparatus shows an obscure charge distribution. Therefore, at first, we tried to improve the spatial resolution of the PEA apparatus. To improve the spatial resolution, it is necessary to reduce a pulse width of applying pulse voltage to generate an acoustic signal wave and/or to reduce the thickness of the detecting piezo-device ${ }^{(3)}$. Therefore, we used both a $4 \mu \mathrm{m}$ thick PVDF (polyviniliden-fluoride) film as a signal detector and a pulse generator for a pulse width of 2 ns. Furthermore, it is 


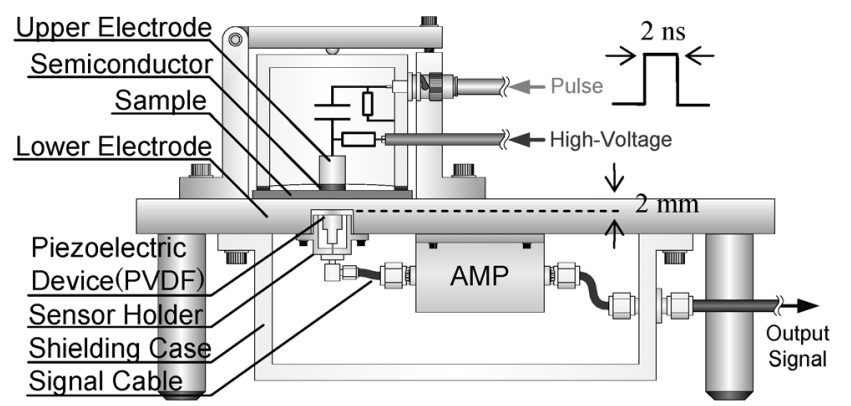

Fig. 1. Improved PEA system with high spatial resolution

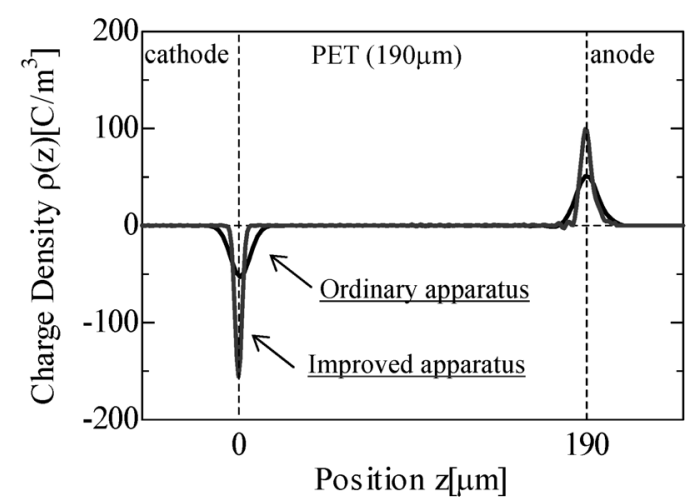

Fig. 2. Comparison between typical signals obtained using the ordinary and the improved apparatus

necessary to reduce the thickness of the lower aluminum electrode because the higher frequency component of the acoustic signal wave is attenuated during passing through the longer distance in lower aluminum electrode ${ }^{(4)}$. Therefore, we use a lower aluminum electrode with thickness of $2 \mathrm{~mm}$. The improved PEA system shows the high spatial resolution of about $5 \mu \mathrm{m}$ for LDPE. Figure 1 shows a schematic measurement system of the developed apparatus. Figure 2 shows a comparison between typical wave forms of the induced charges on electrodes observed using the ordinary and the improved systems. To take them, a PET (Polyethylene Terephthalate) film was used under a DC stress of 2 $\mathrm{kV}$. It is clearly shown that the spatial resolution is improved.

\section{Samples and Experimental Procedure}

Samples used in the experiments are inflation LDPE and LDPE/MgO nano-composite films with thickness of $50-70 \mu \mathrm{m}$. All samples are supplied from J-Power Systems Corp. and they are nominally additive free samples. The $\mathrm{MgO}$ contents in the nano-composite samples are $0.2,0.5$ and $1.0 \mathrm{phr}$. The unit of phr (parts per hundred parts of resin) indicates a rate of weight in gram of $\mathrm{MgO}$ included in $100 \mathrm{~g}$ of LDPE. (For example, $1 \mathrm{phr}$ means that $1 \mathrm{~g}$ of $\mathrm{MgO}$ was mixed to $100 \mathrm{~g}$ of LDPE.) Nano-composite was made by mixing $\mathrm{MgO}$, which has an average diameter of 50 nm, with LDPE by a melt-compounding method. Before the kneading, $\mathrm{MgO}$ was subjected to a surface treatment with a silane coupling agent. Details of LDPE/MgO nano-composite are described elsewhere ${ }^{(1)}$.

Time dependent space charge profiles in the samples under DC electric fields from 100, 200 and $250 \mathrm{kV} / \mathrm{mm}$ at room temperature were measured using the improved apparatus. A DC voltage was applied to the sample using a high voltage amplifier (Matsusada Precision Inc. : HEOPT-20B10) which was able to supply a maximum positive or negative $20 \mathrm{kV}$ by amplifying a signal voltage from PC. In all experiments, the positive DC voltage was applied to the sample using a semi-conducting layer (upper) and an aluminum plate (lower grounded) electrodes system. Since a rapid increase of the high DC voltage applied to the sample sometimes made a breakdown in the sample during the increase of the voltage, we chose a constant value of $500 \mathrm{~V} / \mathrm{s}$ as an initial increase slope of DC voltage to prevent such a breakdown during voltage rising. For example, when we have the space charge profiles in $50 \mu \mathrm{m}$ thick sample under $200 \mathrm{kV} / \mathrm{mm}$ of the DC electric field, we apply $500 \mathrm{~V} / \mathrm{s}$ ramp voltage to the sample and raise it up to $10 \mathrm{kV}$, then we hold the voltage stably. Therefore, it takes $20 \mathrm{~s}$, in the case of above example, from start to stop of raising the voltage. The measurements were carried out with an interval of $10 \mathrm{~s}$ for 1 hour or more unless a breakdown occurred in the sample during the measurement. In fact, any breakdown was not observed in all experiments. All the measurements were carried out on the flesh samples, which was not used for the measurement before. We measured the space charge profiles, at least, three or more times for each experimental condition. For this report, we have chosen the typical results, which seem to show enough reproducibility.

\section{Results and Discussion}

4.1 Space Charge Formation in LDPE Figure 3 (a) shows a typical measurement result of a time dependent space charge distributions in LDPE under an average electric field of $250 \mathrm{kV} / \mathrm{mm}$ for 12 hours. In this figure, the distribution observed at every 1 hour is described. It is clearly shown that a huge amount of positive packet-like charge is injected from the anode into LDPE and it moves towards the cathode side with increasing its amount of charge. However, the movement gradually becomes slow down and finally it seems to be a stable state at 12 hours later from voltage on. Figure 3 (b) shows the electric field distributions that are obtained by calculating the integration of the space charge distributions shown in Fig. 3 (a). It is found that the electric field near the cathode is increasing with increase of the packet-like positive charge. At a stable state, the maximum electric field becomes about $500 \mathrm{kV} / \mathrm{mm}$. All the properties mentioned above about Fig. 3 are almost the same to those observed in our previous report ${ }^{(2)}$. In the previous report, we proposed a model of the generation and the movement of such a packet-like charge. As shown in Fig. 3 (b), the electric field in a zone where the packet-like charge passes through is very low compared with that in a zone ahead of the location of packet-like charge. Since all trap sites in the zone where such a huge charge passed through must be filled by the carriers, the conductivity in the zone should be increased. As mentioned in the report, the packet-like charge may locate at the interface between two zones with different conductivities ${ }^{(2)}$. As shown in Fig. 3 (a), it seems to take about 12 hours to be a stable state. To make it clear, Fig. 4 shows the time dependences of the amount of the packet-like charge and the maximum local electric field. Judging from Fig. 4 , it is clear that the maximum local electric field is gradually saturated to $470 \mathrm{kV} / \mathrm{mm}$. On the other hand, in the previous report, the packet-like charge seems to be the stable state within 10 minutes after voltage on ${ }^{(2)}$. We used an inflation LDPE film as a sample in this experiment, while we used a hot-pressed LDPE film in the previous experiment. Therefore, the difference between this and the previous results may be caused of the difference between morphological structures of the inflation and 


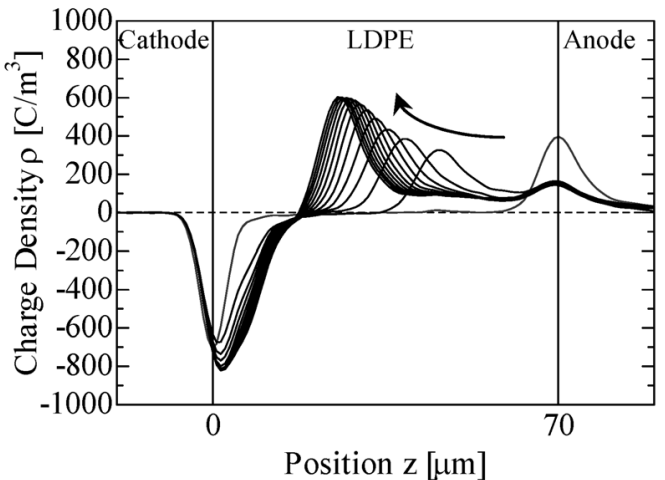

(a) Charge distribution

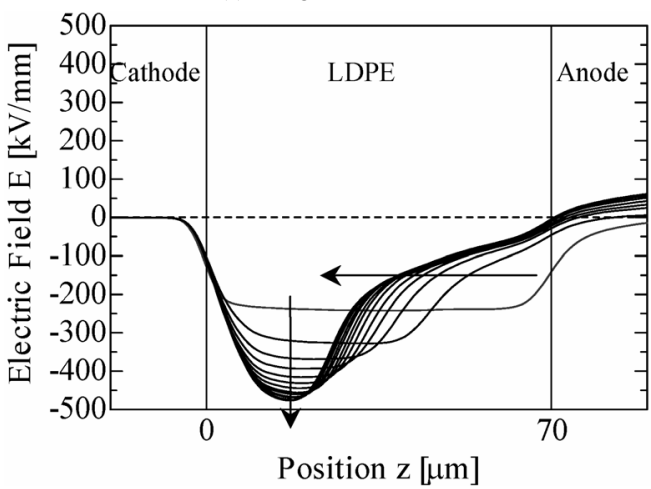

(b) Electric field distribution

Fig. 3. Time dependent (a) charge and (b) electric field distributions in LDPE under electric field of $250 \mathrm{kV} / \mathrm{mm}$ DC stress

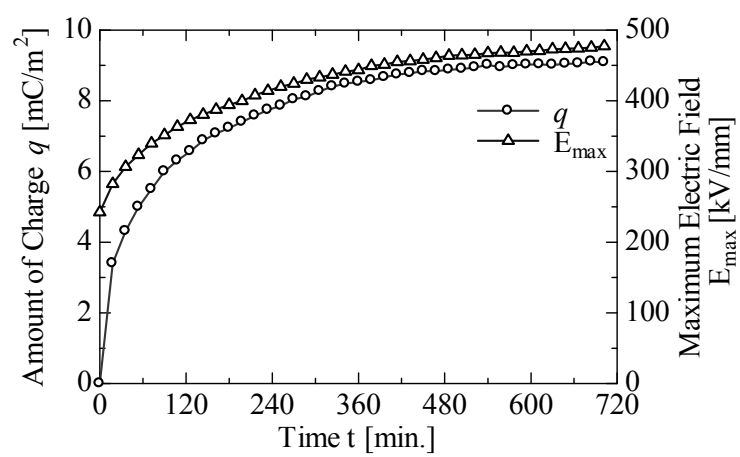

Fig. 4. Time dependent (a) amount of packet-like charge and (b) maximum local electric field in LDPE under electric field of 250 $\mathrm{kV} / \mathrm{mm}$

the hot-pressed films. The slower movement of the packet-like charge is one of remarkable characteristics observed in the inflation LDPE film compared with that observed in the pressed one. Since there are few reports about a comparison between the morphological structures of the inflation and the hot pressed LDPE films, it is difficult to understand the difference of their space charge behavior based on the difference of the morphological structures. However, it can be thought that the inflation film may have a structure like an oriented (elongated) film. An increase of breakdown strength and a decrease of conductivity in LDPE by elongation have been reported by Mita ${ }^{(5)}$ and Masui ${ }^{(6)}$. However, it is difficult to relate the properties to our experimental results directly, since the change of such electrical properties strongly depends on the condition of the elongation. Details should be waited for a future work. Figures 5 (a), (b) and (c) show the time dependent space charge distributions in LDPE observed under applied DC electric field of 100,200 and $250 \mathrm{kV} / \mathrm{mm}$ for 1 hour, respectively. The results are described using a color chart. In the figures, red and blue colors stand for the positive and the negative charge densities, respectively. The broken lines in the figures indicate the position of the cathode (left) and the anode (right) electrodes. Therefore, the colors on the lines mean the existence of the induced charges on electrodes. As shown in Fig. 5 (a), it is found that any packet-like charge is not observed under electric field of 100 $\mathrm{kV} / \mathrm{mm}$. In the previous report, we observed a clear packet-like positive charge injection in LDPE even under electric field of 100 $\mathrm{kV} / \mathrm{mm}$. The difference between this and the previous results may be also caused of the difference of morphological structures between the inflation and the hot-pressed films. As shown in Fig. 5 (b) and (c), the packet-like charge is clearly observed. The packet-like positive charges shown in Fig. 5 (b) and (c) seem to be still moving and increasing even at 1 hour after voltage on. Anyway, when the DC high electric field is applied to a LDPE film, a huge packet-like positive charge appears and it makes the local electric field increase.

\subsection{Space Charge Formation in LDPE/MgO (0.2 phr)}

Figures 6 (a), (b) and (c) show the time dependent space charge distributions in LDPE/MgO nano-composite with $\mathrm{MgO}$ content of $0.2 \mathrm{phr}$ observed under applied DC electric field of 100, 200 and $250 \mathrm{kV} / \mathrm{mm}$ for 1 hour, respectively. As shown in Fig. 6 (a), it isn't found any remarkable packet-like charge under this applied electric field. On the other hand, a complicated behavior was observed in LDPE/MgO (0.2 phr) under applied electric field of $200 \mathrm{kV} / \mathrm{mm}$, as shown in Fig. 6 (b). To show the profiles of the charge and the electric field distributions clearly, some typical distributions at certain times, which are indicated using the white lines in Fig. 6 (b), are described in Fig. 8 (a) and (b). In these results, at first, a relatively small amount of a positive packet-like charge is injected into the bulk and it moves faster towards cathode than that observed in LDPE (i: a result at $1100 \mathrm{~s}$ in Fig. 8). After the packet-like charge reaches to the cathode, a negative charge appears in the middle of bulk, suddenly (ii: a result at 1350 $\mathrm{s}$ in Fig. 8). The negative charge stays at the middle of the bulk with decreasing its amount of charge (iii: a result at $1800 \mathrm{~s}$ in Fig. 8). Then, a new very small positive packet-like charge is injected from the anode and it moves towards the cathode (iv: a result at $2150 \mathrm{~s}$ in Fig. 8). After that, the same process is observed repeatedly. To show the repetitious behavior clearly, Fig. 9 shows a longer observation of the space charge behavior for 3 hours in LDPE $/ \mathrm{MgO}$ with $\mathrm{MgO}$ content of $0.2 \mathrm{phr}$ under applied electric field of $200 \mathrm{kV} / \mathrm{mm}$. A part of this figure is the same as that shown in Fig. 6 (b). To show the repetition clearly, a maximum scale of the color bar in this figure is reduced compared with that in Fig. 6 (b). As shown in Fig. 9, at first, a positive packet-like charge moves across the sample, then a negative charge appears and the repetitious behavior starts. Such a repetitious behavior was often observed in the same condition of the experiments for $\mathrm{LDPE} / \mathrm{MgO}$ with $\mathrm{MgO}$ content of $0.2 \mathrm{phr}$. In the case of $250 \mathrm{kV} / \mathrm{mm}$, while it is not clear in Fig. 6 (c), repetitious behavior similar to that observed in the case of 200 $\mathrm{kV} / \mathrm{mm}$ shown in Fig. 9 was obtained several times in the long time scale observation. The amounts of the positive and the negative charges observed under the electric field of $250 \mathrm{kV} / \mathrm{mm}$ are larger than those observed under the electric field of 200 $\mathrm{kV} / \mathrm{mm}$. As shown in Figs. 6, 8 and 9, the LDPE/MgO 


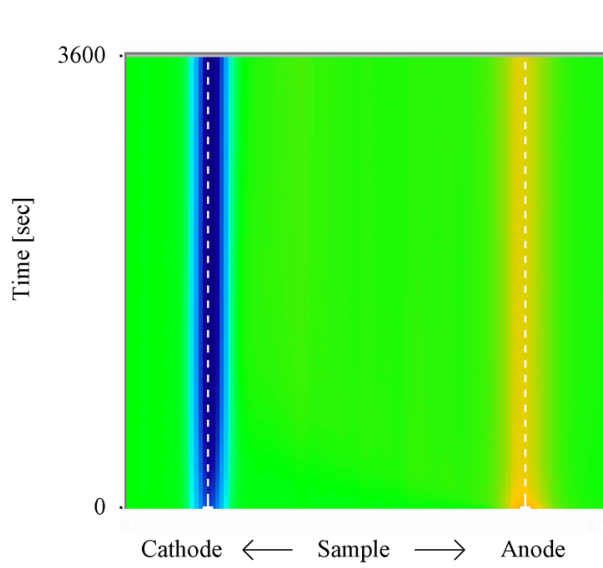

(a) $100 \mathrm{kV} / \mathrm{mm}$ in LDPE

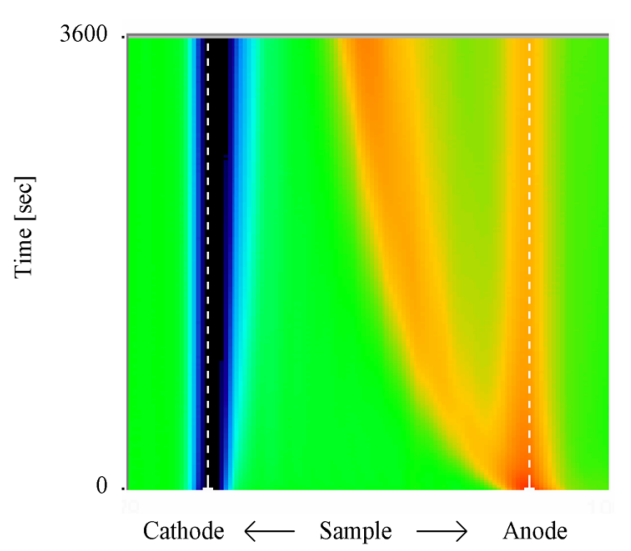

(b) $200 \mathrm{kV} / \mathrm{mm}$ in LDPE

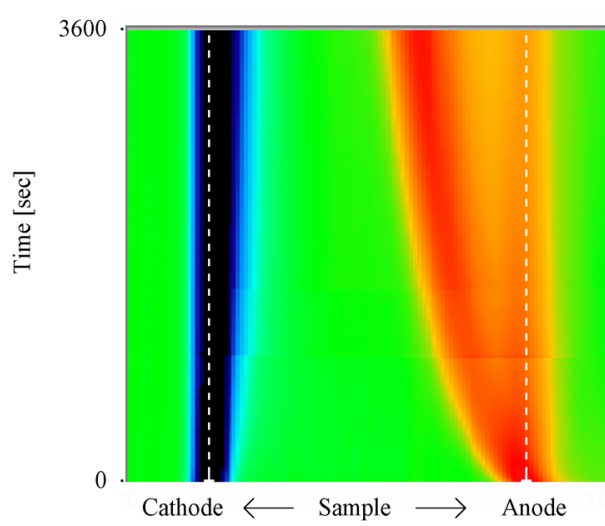

(c) $250 \mathrm{kV} / \mathrm{mm}$ in LDPE

Charge density $\left[\mathrm{C} / \mathrm{m}^{3}\right]$

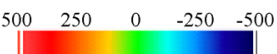

Fig. 5. Time dependent space charge distributions in LDPE under applied electric field of (a) 100, (b) 200 and (c) $250 \mathrm{kV} / \mathrm{mm}$

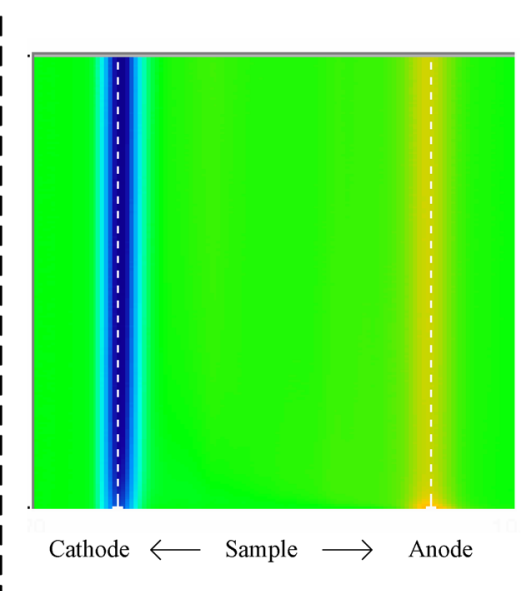

(a) $100 \mathrm{kV} / \mathrm{mm}$ in $\mathrm{LDPE} / \mathrm{MgO}(0.2 \mathrm{phr})$

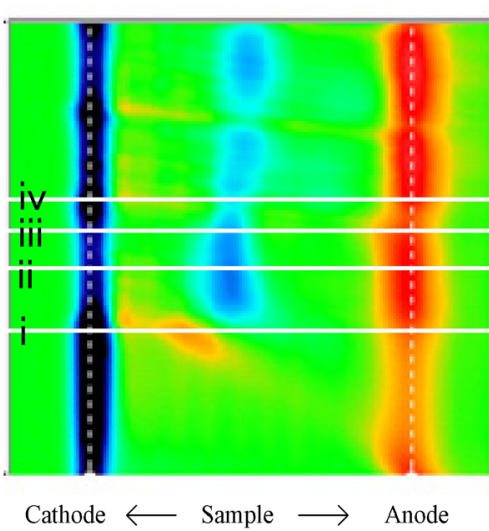

(b) $200 \mathrm{kV} / \mathrm{mm}$ in LDPE/MgO (0.2phr)

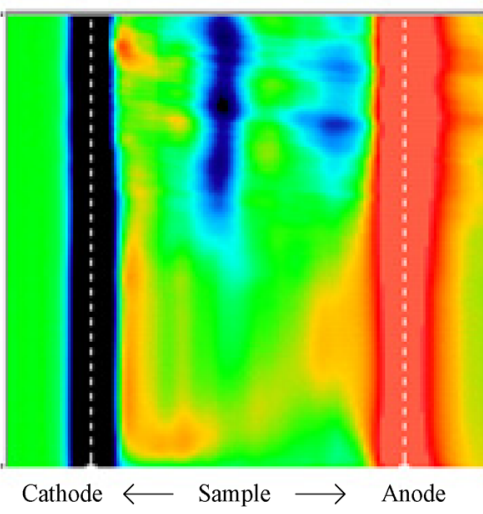

(c) $250 \mathrm{kV} / \mathrm{mm}$ in LDPE/MgO (0.2phr)

Charge density $\left[\mathrm{C} / \mathrm{m}^{3}\right]$

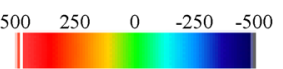

Fig. 6. Time dependent space charge distributions in LDPE/MgO (0.2phr) under applied electric field of (a) 100, (b) 200 and (c) $250 \mathrm{kV} / \mathrm{mm}$

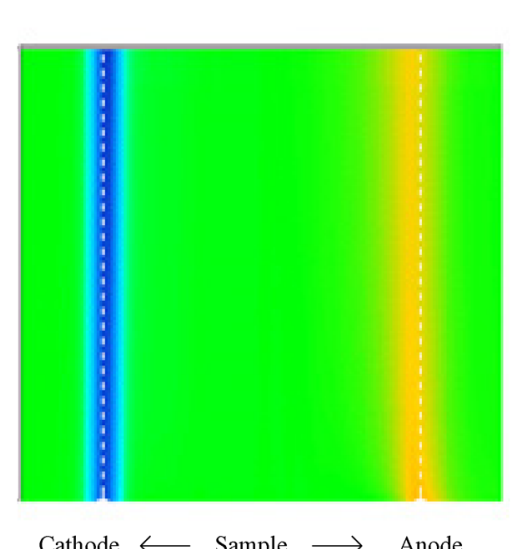

Cathode $\longleftarrow$ Sample $\longrightarrow$ Anode

(a) $100 \mathrm{kV} / \mathrm{mm}$ in $\mathrm{LDPE} / \mathrm{MgO}(1 \mathrm{phr})$

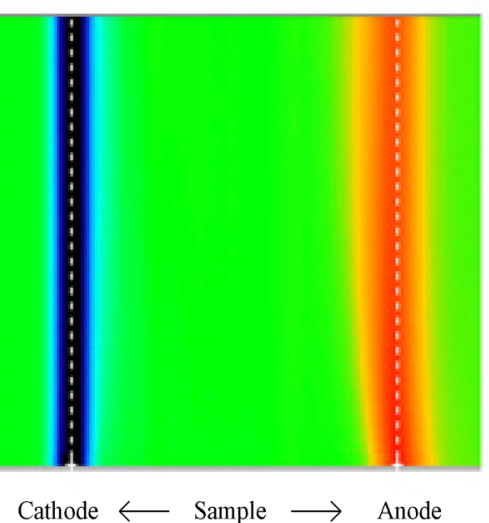

(b) $200 \mathrm{kV} / \mathrm{mm}$ in $\mathrm{LDPE} / \mathrm{MgO}(1 \mathrm{phr})$

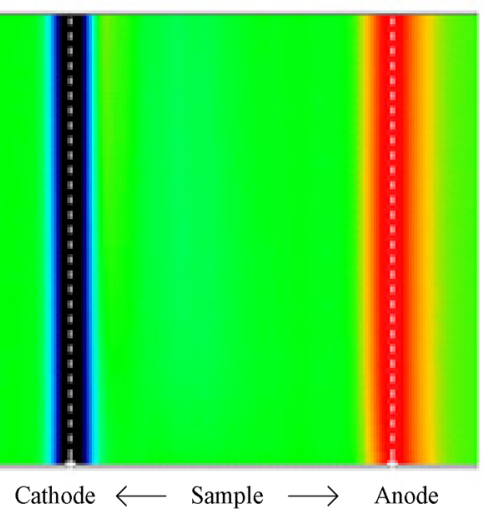

(c) $250 \mathrm{kV} / \mathrm{mm}$ in $\mathrm{LDPE} / \mathrm{MgO}(1 \mathrm{phr})$

Charge density $\left[\mathrm{C} / \mathrm{m}^{3}\right]$ $\begin{array}{rrrrr}500 & 250 & 0 & -250 & -500\end{array}$

Fig. 7. Time dependent space charge distributions in LDPE/MgO (1phr) under applied electric field of (a) 100, (b) 200 and (c) $250 \mathrm{kV} / \mathrm{mm}$ 


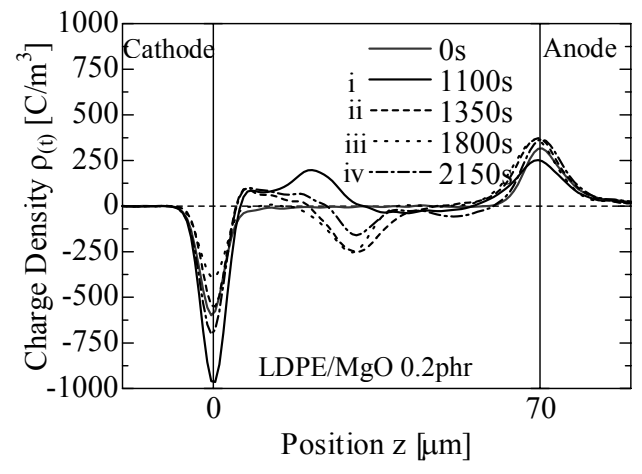

(a) Charge distribution

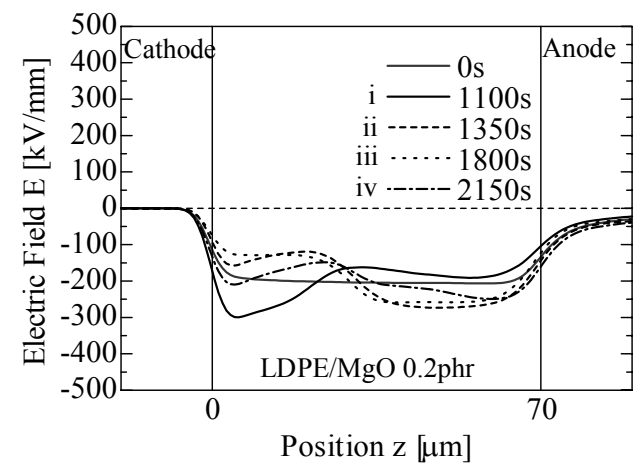

(b) Electric field distribution

Fig. 8. Time dependent (a) charge and (b) electric field distributions in LDPE/MgO (0.2phr) under $200 \mathrm{kV} / \mathrm{mm} \mathrm{DC}$ stress

nano-composite with $\mathrm{MgO}$ content of $0.2 \mathrm{phr}$ shows a complicated performance under high electric field. The repetitions of packet-like charge injection were also observed in some experiments on $\mathrm{XLPE}^{(7)(8)}$. However, any clear explanation has not been given to such phenomena yet. While our proposed model in our previous report suggests the behavior of the positive packet-like charge well, the model is based on a simple assumption of an injection of only positive charge from the anode. However, since the existence of the negative charge was observed in all complicated results, both of the negative and the positive injections and their overlapping or interference must be concerned for the clear explanation of the phenomena. Details of them should be clarified in a future work.

\subsection{Space Charge Formation in LDPE/MgO (1 phr)}

When the space charge distribution in LDPE/MgO (1phr) are observed under electric field of 100,200 and $250 \mathrm{kV} / \mathrm{mm}$, which are shown in Figs. 7 (a), (b) and (c), respectively, it seems to be that almost no remarkable packet-like charge is accumulated in the bulk in all cases. Almost the same results are obtained in observation of the space charge profiles in LDPE/MgO (0.5 phr) under electric field of 100, 200 and $250 \mathrm{kV} / \mathrm{mm}$. However, it seems that a small amount of positive charge is observed at a vicinity of the cathode electrode as shown in Fig. 7 (c). It is difficult to recognize the movement of the positive charge from Fig. 7, however, by careful observation, we can find that a very small packet-like charge moves from the anode to the cathode quickly. However, since the amount of charge is very small, the electric field doesn't seem to be affected by the positive charge. Therefore, it can be said that no remarkable packet-like charge generates in LDPE/MgO nano-composite with $\mathrm{MgO}$ content of

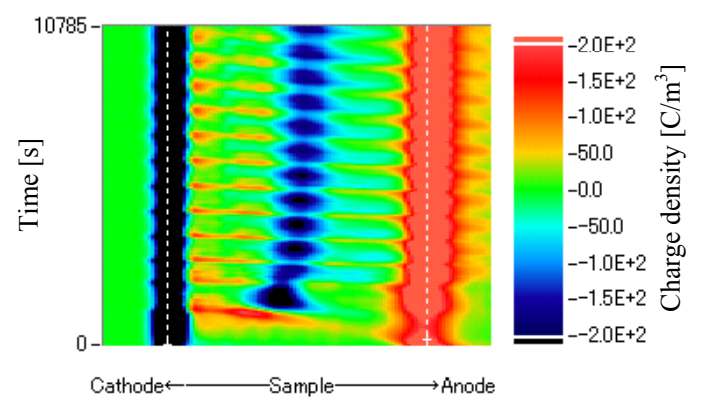

Fig. 9. Space charge distribution in LDPE/MgO (0.2 phr) under electric field of $200 \mathrm{kV} / \mathrm{mm}$ for about 3 hours

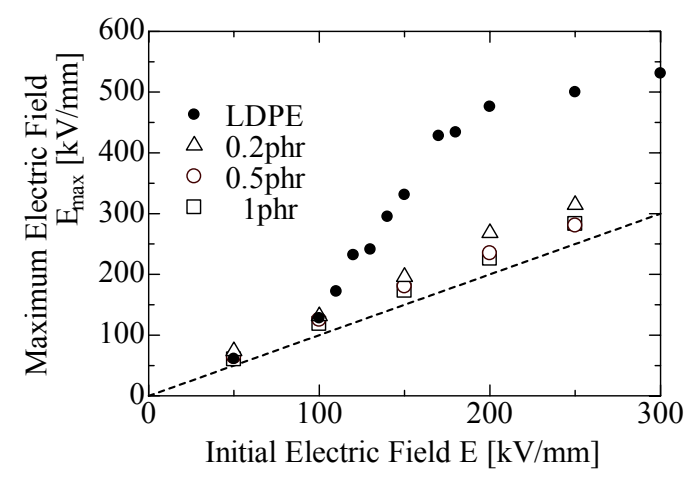

Fig. 10. Relationship between initial and local maximum electric fields

more than $0.5 \mathrm{phr}$ under the applied electric field up to 250 $\mathrm{kV} / \mathrm{mm}$.

4.4 Enhancement of Electric Field Figure 10 shows a relationship between the initial applied and the local maximum electric fields in the samples with various content of $\mathrm{MgO}$. All the data are averaged from three or more experimental results. The maximum local electric fields are calculated from the charge distributions that seem to be the stable states. Therefore, some of them in LDPE are obtained at more than 10 hours after voltage on. However, in the case of LDPE/MgO, since the space charge distributions seem to be stable or repeating same cycles within 1 hour after voltage on, the data are maximum values in the distributions within 1 hour. In this figure, the broken line indicates the applied electric field. Therefore, the data over the line mean that the electric fields are enhanced by the space charges in the bulk. When the initial electric field of more than $100 \mathrm{kV} / \mathrm{mm}$ is applied to LDPE film, the local electric field is strongly enhanced. Especially in the case of more than 200 $\mathrm{kV} / \mathrm{mm}$, the enhanced electric field reaches to about $500 \mathrm{kV} / \mathrm{mm}$. On the other hand, the enhancement of the local electric field in LDPE $/ \mathrm{MgO}$ is very small compared with those in LDPE. Especially in the case of LDPE/MgO with $\mathrm{MgO}$ content of more than $0.5 \mathrm{phr}$, the enhancement is tiny even under $250 \mathrm{kV} / \mathrm{mm}$ of the initial applied field.

\subsection{Effect of $\mathrm{MgO}$ on Reduce of Charge Injection}

Finally, the effect of $\mathrm{MgO}$ on reduce of charge injection in $\mathrm{LDPE} / \mathrm{MgO}$ composite is concerned. As mentioned above, a very small amount of the positive packet-like charge is observed in $\mathrm{LDPE} / \mathrm{MgO}$ nano-composite including $\mathrm{MgO}$ more than $0.5 \mathrm{phr}$. Therefore, it can be thought that the behavior of the injected positive packet-like charge changed to be quicker and smaller compared with that in LDPE. According to the previously 
proposed model of the packet-like charge behavior ${ }^{(2)}$, there seems to be a reasonable explanation for the phenomena. When the injection barrier increases, the amount of injected packet-like charge is expected to be decreased and consequently the speed of packet-like charge is expected to be decreased. If the $\mathrm{MgO}$ plays as deep traps for the carriers, the injection barrier may increase because many carriers are captured at the interface between the electrode and the sample. However, since any clear evidence for the above explanation has been not found yet, the future work is expected to make it clear.

\section{Conclusion}

Space charge formation in LDPE/MgO nano-composite thin films under a high DC electric field of more than $100 \mathrm{kV} / \mathrm{mm}$ were studied using an improved PEA system with a high spatial resolution. In LDPE, it was found that a huge amount of positive packet-like charge was injected into the bulk and it made a local electric field higher. On the other hand, there was almost no remarkable space charge was observed in the LDPE/MgO nano-composite film with $\mathrm{MgO}$ content of more than $0.5 \mathrm{phr}$. In the case of LDPE/MgO with $\mathrm{MgO}$ content of $0.2 \mathrm{phr}$, a complicated repetition of a small packet-like charge was observed. It is found that even such a small amount of $\mathrm{MgO}$ content of 0.5 $\mathrm{phr}$ in the nano-composite is effective to prevent the charge injection which is caused of the enhancement of the local electric field. This research work was party supported by a Grant-in-Aid for Scientific Research (c) (No. 18560315) from the Ministry of Education, Science, Sports and Culture Japan.

(Manuscript received Feb. 6, 2006, revised June 20, 2006)

\section{References}

(1) Y. Murata, M. Kanaoka, and S. Katakai : "Effect of Nano-sized Filler on Breakdown Strength in HVDC Cable", IEEJ Trans. FM, Vol.126, No.11, pp.00-00 (2006)

(2) K. Matsui, Y. Tanaka, T. Takada, T. Fukao, K. Fukunaga, T. Maeno, and J. M. Alison : "Space Charge Behavior in Low-density Polyethylene at Pre Breakdown", IEEE Trans. DEI, Vol.12, No.3, pp.406-415 (2005)

(3) T. Takada, Y. Tanaka, N. Adachi, and X. Qin : "Comparison Between the PEA Method and the PWP Method for Space Charge Measurement in Solid Dielectrics", IEEE Trans. DEI, Vol.5, No.6, pp.944-951 (1998)

(4) Y. Li, M. Aihara, K. Murata, Y. Tanaka, and T. Takada : "Space Charge Measurement in Thick Dielectric Materials by Pulsed Electroacoustic Method", Rev. Sci. Instr., Vol.66, No.7 (1995)

(5) S. Mita and K. Yahagi : "Effect of Elongation on Dielectric Breakdown Strength in Polyethylene", Jap. J. Appl. Phys., Vol.14, No.2, pp.197-201 (1975)

(6) M. Masui, H. Nagasawa, and K. Yahagi : "Effect of Uniaxial Elogation on Electrical Conduction in Low Density Polyethylene", T. IEE Japan, Vol.188-A, No.4, pp.187-194 (1982) (in Japanese)

(7) N. Hozumi, T. Takeda, H. Suzuki, and T. Okamoto : "Space Charge Behavior in XLPE Cable Insulation under 0.2-1.2 MV/cm dc Field", IEEE Trans DEI, Vol.5, No.1, pp.82-90 (1998)

(8) K. Matsui, Y. Tanaka, T. Takada, and T. Maeno : "Space Charge Observation in Various Types of Polyethylene under Ultra-high DC Electric Field", Proc. 2004 IEEE International Conference on Solid Dielectrics, (8th ICSD), Vol.1, pp.201-204 (2004)

Yuji Hayase

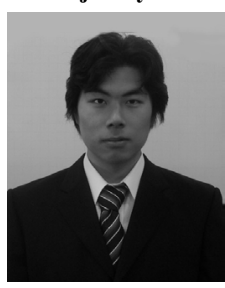

(Student Member) was born in Hokkaido, Japan in 1983. He is a graduate student of Master of Engineering course in Mechanical Systems Engineering, Musashi Institute of Technology. $\mathrm{He}$ has been involved in space charge behavior in polymers.

Hiroyuki Aoyama

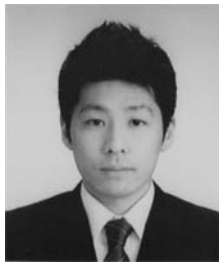

(Non-member) was born in Tokyo, Japan in 1980. received the B.E and M.E degrees in mechanical systems engineering from Musashi Institute of Technology, Japan, in 2004 and 2006, respectively. He joined Agilent Technologies Japan, Ltd. in 2006.

Kohei Matsui

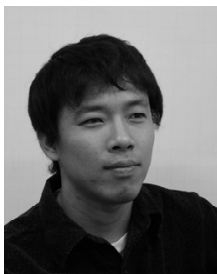

module.

(Non-member) was born in Hiroshima, Japan in 1978. $\mathrm{He}$ received the B.E, M.E and Ph. D. degrees in mechanical systems engineering from Musashi Institute of Technology, Japan, in 2001, 2003 and 2006, respectively. He was a visiting student at University of Southampton from 2003 to 2004. He joined Fuji Electric Advanced Technology Co., Ltd. in 2006, and he is involved to develop the insulation system for power

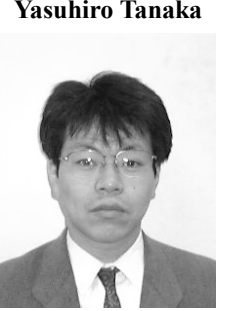

(Member) was born in Fukuoka, Japan in 1961. He received the B.E., the M.E. and Ph. D. degrees in electrical engineering from Waseda University, Japan, in1986, 1988 and 1991, respectively. He became a Lecturer, an Associate Professor and a Professor at Musashi Institute of Technology, in 1992, 1998 and 2004, respectively. He was a Visiting Scientist at University of Southampton from 1999 to 2000. Currently, he is developing the measurement system for the space charge distribution in various solid dielectric materials at high temperature, under ultra-high electric field or under irradiation of gamma-ray or electron-beam in vacuum.

Tatsuo Takada

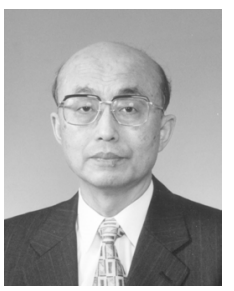

(Member) was born in Yamanashi, Japan in 1939. He received the B.E. degree in electrical engineering from Muashi Institute of Technology, Japan, in 1963 and the M.E. and Ph. D. degree from Tohoku University, Japan in 1966 and 1975, respectively. He became a Lecturer, an Associate Professor and a Professor at Musashi Institute of Technology at Musashi Institute of Technology, respectively in 1967, 1974 and 1987 . He was a Visiting Scientist at MIT (USA) from 1981 to 1983 . He received the Excellent Paper Award from the IEE of Japan in 1974, 1981 and 1990. In 1990, Dr. Takada received the Whitehead Memorial Lectureship at the IEEE CEIDP. Currently, he is involved with several research projects on the space charge effects in solid dielectric materials, surface charges on thin-film, and electric field measurements in liquid materials.

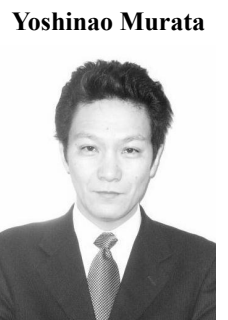

(Member) was received a B. S. degree in physics from the Science University of Tokyo in 1992. In the same year, he joined Hitachi Cable Ltd. Since then, he has been engaged in research and development of insulating materials for power cables. In 2001, he moved to J-power systems Corporation and is presently a researcher of the Research \& Development Center of the company. 